Supporting Information-1

\title{
Enantioselective Total Synthesis of (+)-Jasplakinolide
}

\author{
Arun K. Ghosh,* and Deuk Kyu Moon \\ Departments of Chemistry and Medicinal Chemistry, \\ Purdue University, West Lafayette, IN 47907
}

\section{General}

${ }^{1} \mathrm{H}$ NMR and ${ }^{13} \mathrm{C}$ NMR spectra were recorded on Bruker Avance DRX-400 spectrometers. IR spectra were recorded on a Mattason Genesis II FT-IR spectrometer. Optical rotations were recorded on a Perkin Elmer 341 polarimeter. Anhydrous solvents were obtained as follows: THF and diethyl ether by distillation from sodium and benzophenone; dichloromethane from $\mathrm{CaH}_{2}$. All other solvents were reagent grade. All moisture sensitive reactions were carried out in flame dried flask under nitrogen atmosphere. Column chromatography was performed with Whatman 240-400 mesh silica gel. TLC was carried out with E. Merck silica gel 60-F-254 plates.

\section{Experimental Section}

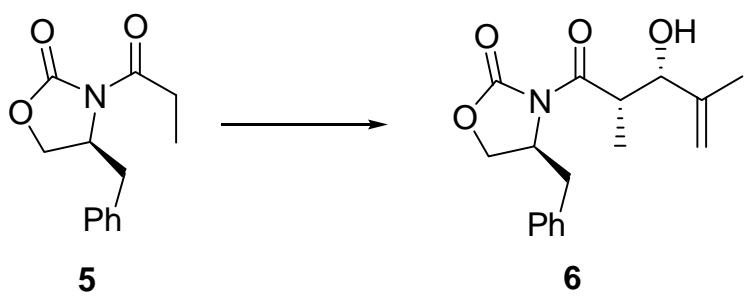

To a stirred solution of oxazolidinone $5(13.5 \mathrm{~g}, 57.9 \mathrm{mmol})$ in $\mathrm{CH}_{2} \mathrm{Cl}_{2}(200 \mathrm{~mL})$ was added $\mathrm{Bu}_{2} \mathrm{BOTf}(69.5 \mathrm{~mL}, 69.5 \mathrm{mmol})$ and diisopropylethylamine $(14 \mathrm{~mL}, 81 \mathrm{mmol})$ at $0{ }^{\circ} \mathrm{C}$. After stirring for $50 \mathrm{~min}$, distilled methacrolein $(12 \mathrm{~mL}, 144.7 \mathrm{mmol})$ was added to the enolate 
reaction mixture at $-78{ }^{\circ} \mathrm{C}$. The reaction mixture was stirred for $20 \mathrm{~min}$ and then was warmed to $0{ }^{\circ} \mathrm{C}$. After additional $1 \mathrm{~h}, \mathrm{pH} 7$ buffer solution $(10 \mathrm{~mL})$ followed by $\mathrm{MeOH}(20 \mathrm{~mL})$ was added to the reaction mixture at $0{ }^{\circ} \mathrm{C}$ and continuously added $\mathrm{H}_{2} \mathrm{O}_{2}(20 \mathrm{~mL}), \mathrm{MeOH}(20 \mathrm{~mL})$ at the same temperature. After stirring for $1 \mathrm{~h}$ at $0{ }^{\circ} \mathrm{C}$, a volatile solvent was removed and then $\mathrm{H}_{2} \mathrm{O}$ was added to the reaction mixture. The mixture was extracted with EtOAc. The combined extracts were dried over anhydrous $\mathrm{MgSO}_{4}$, filtered, and concentrated in vacuo. The organic residue was purified by column chromatography on silica gel (25\% ethyl acetate in hexane) to afford $16.5 \mathrm{~g}$ (54.4 mmol, 94\%) of 6. ${ }^{1} \mathrm{H} \mathrm{NMR}\left(400 \mathrm{MHz}, \mathrm{CDCl}_{3}\right) \delta$ 7.34-7.30(m, $\left.2 \mathrm{H}\right)$, 7.277.24(m, $1 \mathrm{H}), 7.19-7.18(\mathrm{~m}, 2 \mathrm{H})$, 5.10(s, $1 \mathrm{H}), 4.96(\mathrm{~s}, 1 \mathrm{H}), 4.71-4.67(\mathrm{~m}, 1 \mathrm{H}), 4.40(\mathrm{~s}, 1 \mathrm{H})$, 4.24-4.16(m, 2 H), 3.96-3.92(m, 1 H), $3.25(\mathrm{dd}, J=13.4 \mathrm{~Hz}, 3.3 \mathrm{~Hz}, 1 \mathrm{H}) 2.77(\mathrm{dd}, J=13.4 \mathrm{~Hz}$, $9.4 \mathrm{~Hz}, 1 \mathrm{H}), 2.68(\mathrm{bs}, 1 \mathrm{H}), 1.71(\mathrm{~s}, 3 \mathrm{H}), 1.17(\mathrm{~d}, J=7.1 \mathrm{~Hz}, 3 \mathrm{H}) ;{ }^{13} \mathrm{C} \mathrm{NMR}\left(100 \mathrm{MHz}, \mathrm{CDCl}_{3}\right)$ $\delta 177.1,152.9,143.7,135.0,129.4,128.9,127.4,111.8,73.9,66.2,55.2,40.1,37.7,19.4,9.99$
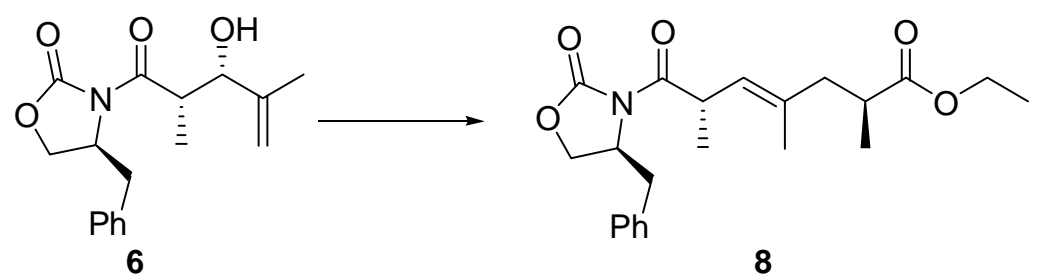

To a solution of aldol adduct $6(8 \mathrm{~g}, 26.4 \mathrm{mmol})$ was added propionic acid $(0.4 \mathrm{~mL}$, $5.28 \mathrm{mmol}$ ) and triethylorthopropionate $(50 \mathrm{~mL})$. The reaction mixture was stirred for $1 \mathrm{~h}$ at 140 ${ }^{\circ} \mathrm{C}$. The reaction mixture was cooled to room temperature and was purified directly by column chromatography on silica gel (10\% ethyl acetate in hexane) to afforded $9.5 \mathrm{~g}(24.6 \mathrm{mmol}, 93 \%)$ of $8,[\alpha]_{\mathrm{D}}^{23}=+104, c 1.0, \mathrm{CHCl}_{3}$; IR (neat) 1782, 1730, $1699 ;{ }^{1} \mathrm{H} \mathrm{NMR}\left(400 \mathrm{MHz}, \mathrm{CDCl}_{3}\right) \delta$ 7.33-7.29(m, 2 H), 7.27-7.25(m, 1 H), 7.20-7.18(m, 2 H), 5.28(d, J = 8.5 Hz, 1 H), 4.64-4.60(m, 2 H), 4.16-4.12(m, 2 H), 4.11-4.07(m, 2 H), 3.26(dd, $J=13.4 \mathrm{~Hz}, 3.2 \mathrm{~Hz}, 1 \mathrm{H}), 2.75(\mathrm{dd}, J=$ $13.4 \mathrm{~Hz}, 9.7 \mathrm{~Hz}, 1 \mathrm{H}), 2.63-2.57(\mathrm{~m}, 1 \mathrm{H}), 2.39-2.34(\mathrm{~m}, 1 \mathrm{H}), 2.08-2.05(\mathrm{~m}, 1 \mathrm{H}), 1.65(\mathrm{~d}, J=1.2$ $\mathrm{Hz}, 3 \mathrm{H}), 1.26-1.21(\mathrm{~m}, 6 \mathrm{H}), 1.07(\mathrm{~d}, J=6.9 \mathrm{~Hz}, 3 \mathrm{H}) ;{ }^{13} \mathrm{C} \mathrm{NMR}\left(100 \mathrm{MHz}, \mathrm{CDCl}_{3}\right) \delta 176.2$, 175.7, 152.9, 135.3, 135.2, 129.4, 128.9, 127.3, 125.6, 65.9, 60.1, 55.5, 43.7, 37.9, 37.8, 37.2, 18.2, 16.5, 16.2, $14.2 ;$ LRMS(ESI) $\mathrm{m} / \mathrm{z}[\mathrm{M}+\mathrm{Na}]^{+}$410.11, HRMS(ESI) $[\mathrm{M}+\mathrm{Na}]^{+}$: calculated for $\mathrm{C}_{22} \mathrm{H}_{29} \mathrm{NO}_{5}: 410.1943$, Found : 410.1942 . 


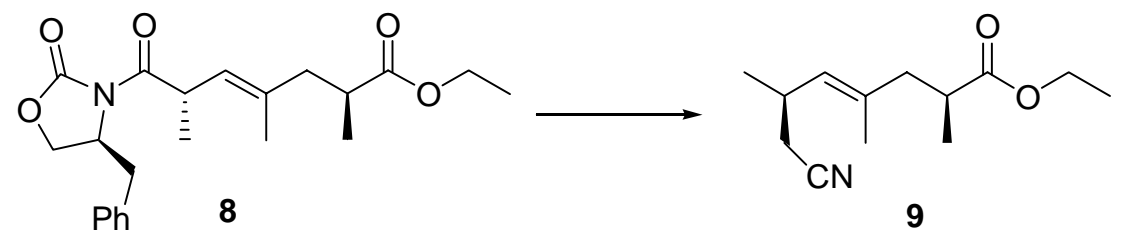

To a solution of Claisen adduct $8(8.5 \mathrm{~g}, 22 \mathrm{mmol})$ in $\mathrm{Et}_{2} \mathrm{O}(70 \mathrm{~mL})$ was added $\mathrm{EtOH}$ $(1.3 \mathrm{~mL}, 22 \mathrm{mmol})$ and $\mathrm{LiBH}_{4}\left(10.5 \mathrm{~mL}, 21 \mathrm{mmol}: 2 \mathrm{M}\right.$ in THF) at $0{ }^{\circ} \mathrm{C}$. After stirring for 20 min. sodium-potaisum tartrate (sat.) was added to the reaction mixture and then was extracted with EtOAc. The organic layer was dried over anhydrous $\mathrm{MgSO}_{4}$, filtered, and concentrated in vacuo, the residue was purified by column chromatography (20\% ethyl acetate in hexane) to afford $3.9 \mathrm{~g}$ (18.2 $\mathrm{mmol}, 83 \%)$ of the corresponding alcohol.

To the solution of alcohol (30 mg, $0.14 \mathrm{mmol})$ in THF $(2 \mathrm{~mL})$ was added triphenylphosphine (73 mg, $0.28 \mathrm{mmol})$ and diisopropylazodicarboxylate $(55 \mu \mathrm{L}, 0.28 \mathrm{mmol})$ dropwise at $0{ }^{\circ} \mathrm{C}$. After stirring for $10 \mathrm{~min}$ at ice bath, acetone cyanohydrin $(20 \mu \mathrm{L}, 0.21 \mathrm{mmol})$ was added to the reaction mixture. The resulting mixture was allowed to warm to reflux and then stirred for $6 \mathrm{~h}$. The reaction mixture was poured into $\mathrm{H}_{2} \mathrm{O}$. The resulting solution was extracted with EtOAc and the combined organic extracts were dried over anhydrous $\mathrm{MgSO}_{4}$, filtered, and concentrated in vacuo. The residue was purified by flash chromatography $(10 \%$ ethyl acetate in hexane) to afford $24 \mathrm{mg}(0.11 \mathrm{mmol}, 79 \%)$ of $9,[\alpha]_{\mathrm{D}}{ }^{23}=+6.1, c 3.0, \mathrm{CHCl}_{3}$; IR (neat) 2246, $1732 ;{ }^{1} \mathrm{H}$ NMR $\left(400 \mathrm{MHz}, \mathrm{CDCl}_{3}\right) \delta 4.97(\mathrm{~d}, J=9.6 \mathrm{~Hz}, 1 \mathrm{H}), 4.09-4.02(\mathrm{~m}, 2 \mathrm{H})$, 2.77-2.71(m, $1 \mathrm{H}), 2.58-2.53(\mathrm{~m}, 1 \mathrm{H}), 2.35-2.30(\mathrm{~m}, 1 \mathrm{H}), 2.23-2.20(\mathrm{~m}, 2 \mathrm{H}), 2.03-1.97(\mathrm{~m}, 1 \mathrm{H})$, $1.61(\mathrm{~d}, J=1.0 \mathrm{~Hz}, 3 \mathrm{H}), 1.19(\mathrm{t}, 3 \mathrm{H}), 1.07(\mathrm{~d}, J=6.8 \mathrm{~Hz}, 3 \mathrm{H}), 1.02(\mathrm{~d}, J=6.8 \mathrm{~Hz}, 3 \mathrm{H}) ;{ }^{13} \mathrm{C}$ NMR $\left(100 \mathrm{MHz}, \mathrm{CDCl}_{3}\right) \delta 176.1,134.4,129.1,118.6,60.1,43.6,37.7,29.7,24.8,20.3,16.5$, 15.9, 14.1; LRMS(ESI) $\mathrm{m} / \mathrm{z} \quad[\mathrm{M}+\mathrm{Na}]^{+}$245.88, HRMS(ESI) $[\mathrm{M}+\mathrm{Na}]^{+}:$calculated for $\mathrm{C}_{13} \mathrm{H}_{21} \mathrm{NO}_{2}: 246.1470$, Found : 246.1468 .<smiles>CCOC(=O)C(C)CC(C)=CC(C)C[C@@H](C)CC(C)=CC(C)C[C@@H](C)OCC</smiles> 
To a solution of nitrile 9 (38 mg, $0.17 \mathrm{mmol})$ in pyridine / $\mathrm{AcOH} / \mathrm{H}_{2} \mathrm{O}(0.4 \mathrm{~mL} / 0.2$ $\mathrm{mL} / 0.2 \mathrm{~mL}$ ) was added hydrated sodium hypophosphite $\mathrm{NaH}_{2} \mathrm{PO}_{3} \cdot \mathrm{H}_{2} \mathrm{O}(90 \mathrm{mg}, 0.85 \mathrm{mmol})$ and $50 \mathrm{mg}$ of Raney-Ni (50\% slurry in water). The reaction mixture was stirred for $3 \mathrm{~h}$ at $50{ }^{\circ} \mathrm{C}$, $50 \mathrm{mg}$ of Raney-Ni was added, and the mixture was stirred for additional $3 \mathrm{~h}$. The mixture was filtered and then the catalyst was washed with water. The mixture was extracted with $\mathrm{Et}_{2} \mathrm{O}$, dried over anhydrous $\mathrm{MgSO}_{4}$, filtered and the resulting solution was concentrated in vacuo. The organic residue was purified by column chromatography on silica gel (7\% ethyl acetate in hexane) to afford $31 \mathrm{mg}(0.14 \mathrm{mmol}, 81 \%)$ of aldehyde.

A solution of aldehyde (31 mg, $0.14 \mathrm{mmol})$ in THF $(1 \mathrm{~mL})$ was treated with $\mathrm{MeMgBr}$ $\left(53 \mu \mathrm{L}, 0.16 \mathrm{mmol}, 3 \mathrm{M}\right.$ in THF) at $0{ }^{\circ} \mathrm{C}$ and was stirred for $30 \mathrm{~min}$. The reaction mixture was poured into $\mathrm{NH}_{4} \mathrm{Cl}$ (sat.) and the aqueous layer was extracted with EtOAc. The combined organic extracts were dried over anhydrous $\mathrm{MgSO}_{4}$, concentrated in vacuo and purified by the flash chromatography on silica gel ( $15 \%$ ethyl acetate in hexane) to produce $30 \mathrm{mg}(0.12 \mathrm{mmol}$, $90 \%)$ of alcohol 10, $11 ; \mathbf{1 0},{ }^{1} \mathrm{H}$ NMR $\left(400 \mathrm{MHz}, \mathrm{CDCl}_{3}\right) \delta 4.88(\mathrm{~d}, J=9.8 \mathrm{~Hz}, 1 \mathrm{H}), 4.10-$ 4.04(m, $2 \mathrm{H}), 3.71-3.67(\mathrm{~m}, 1 \mathrm{H}), 2.60-2.55(\mathrm{~m}, 2 \mathrm{H}), 2.32(\mathrm{dd}, J=13.7 \mathrm{~Hz}, 7.5 \mathrm{~Hz}, 1 \mathrm{H}), 2.00(\mathrm{dd}$, $J=13.7 \mathrm{~Hz}, 7.5 \mathrm{~Hz}, 1 \mathrm{H}), 1.61(\mathrm{~d}, J=0.9 \mathrm{~Hz}, 3 \mathrm{H}), 1.53(\mathrm{bs}, 1 \mathrm{H}), 1.40-1.37(\mathrm{~m}, 1 \mathrm{H}), 1.28-$ 1.24(m, $1 \mathrm{H}), 1.22(\mathrm{t}, 3 \mathrm{H}) 1.13(\mathrm{~d}, J=6.2 \mathrm{~Hz}, 3 \mathrm{H}), 1.07(\mathrm{~d}, J=6.9 \mathrm{~Hz}, 3 \mathrm{H}), 0.88(\mathrm{~d}, J=6.8 \mathrm{~Hz}$, $3 \mathrm{H}) ;{ }^{13} \mathrm{C} \mathrm{NMR}\left(100 \mathrm{MHz}, \mathrm{CDCl}_{3}\right) \delta 176.6,133.0,131.6,66.3,60.1,47.0,43.9,38.0,29.1$, $24.1,21.6,16.7,15.9,14.2$

11, $[\alpha]_{\mathrm{D}}{ }^{23}=-27.9, c 1.3, \mathrm{CHCl}_{3}$; IR (neat) 3446,$1734 ;{ }^{1} \mathrm{H}$ NMR $\left(400 \mathrm{MHz}, \mathrm{CDCl}_{3}\right) \delta 4.98(\mathrm{~d}, J$ $=9.6 \mathrm{~Hz}, 1 \mathrm{H}), 4.10-4.04(\mathrm{~m}, 2 \mathrm{H}), 3.79-3.74(\mathrm{~m}, 1 \mathrm{H}), 2.60-2.55(\mathrm{~m}, 1 \mathrm{H}), 2.49-2.41(\mathrm{~m}, 1 \mathrm{H})$, 2.34-2.29(m, $1 \mathrm{H}), 2.02-1.97(\mathrm{~m}, 1 \mathrm{H}), 1.66(\mathrm{bs}, 1 \mathrm{H}), 1.60(\mathrm{~d}, J=1.1 \mathrm{~Hz}, 3 \mathrm{H}), 1.41-1.36(\mathrm{~m}, 2 \mathrm{H})$, $1.22(\mathrm{t}, 3 \mathrm{H}), 1.12(\mathrm{~d}, J=6.2 \mathrm{~Hz}, 3 \mathrm{H}), 1.07(\mathrm{~d}, J=6.9 \mathrm{~Hz}, 3 \mathrm{H}), 0.89(\mathrm{~d}, J=6.8 \mathrm{~Hz}, 3 \mathrm{H}) ;{ }^{13} \mathrm{C}$ NMR $\left(100 \mathrm{MHz}, \mathrm{CDCl}_{3}\right) \delta 176.4,133.4,131.4,67.1,60.1,47.0,43.9,38.0,30.2,23.4,21.3$, 16.7, 15.9, $14.2 ;$ LRMS(ESI) $\mathrm{m} / \mathrm{z}[\mathrm{M}+\mathrm{Na}]^{+}$242.82, HRMS(ESI) $[\mathrm{M}+\mathrm{Na}]^{+}$: calculated for $\mathrm{C}_{14} \mathrm{H}_{26} \mathrm{O}_{3}: 243.1960$, Found : 243.1959. 


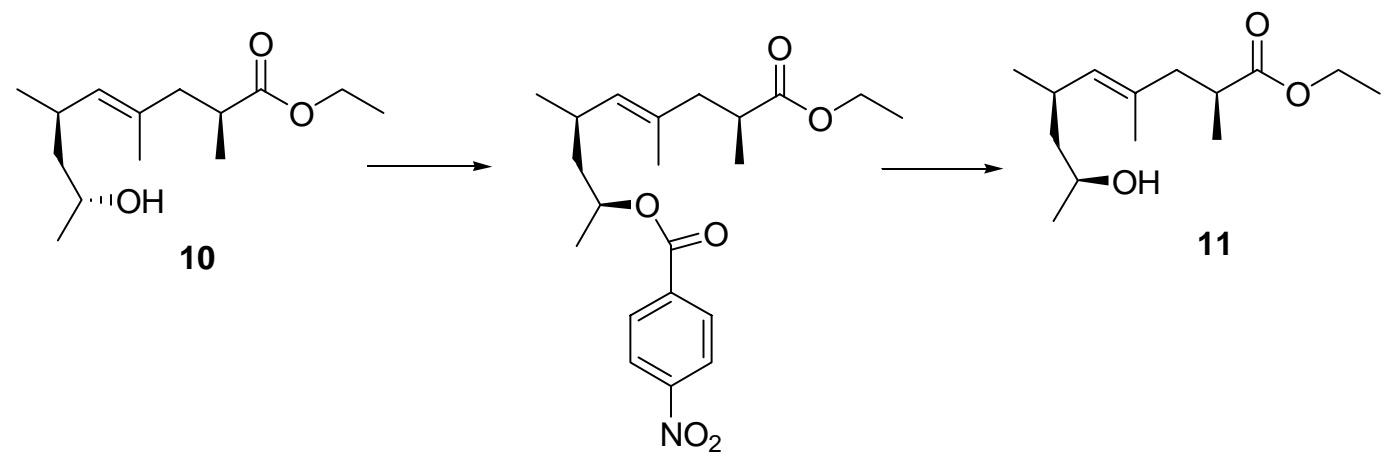

To a solution of (R)-alcohol 10 (10 mg, $0.04 \mathrm{mmol})$ in $\mathrm{Et}_{2} \mathrm{O}(3 \mathrm{~mL})$ was added $\mathrm{Ph}_{3} \mathrm{P}(108$ $\mathrm{mg}, 0.4 \mathrm{mmol}), p$-nitrobenzoic acid $(69 \mathrm{mg}, 0.4 \mathrm{mmol})$ and then diisopropylazodi carboxylate $(40 \mu \mathrm{L}, 0.2 \mathrm{mmol})$ dropwise at room temperature. After stirring for $10 \mathrm{~h}$, the reaction mixture was filtered and concentrated in vacuo.

To the solution of crude compound in $\mathrm{EtOH}$ was added potassium carbonate at room temperature. After stirring for $5 \mathrm{~h}$, the reaction mixture was quenched with $\mathrm{NH}_{4} \mathrm{Cl}$ (sat) and extracted with EtOAc. The organic extracts were dried over anhydrous $\mathrm{MgSO}_{4}$, filtered, and concentrated in vacuo. Flash chromatography on silica gel (15\% ethyl acetate in hexane) produced $7.8 \mathrm{mg}(0.03 \mathrm{mmol}, 78 \%)$ of $(\mathrm{S})-\mathbf{1 1}$.

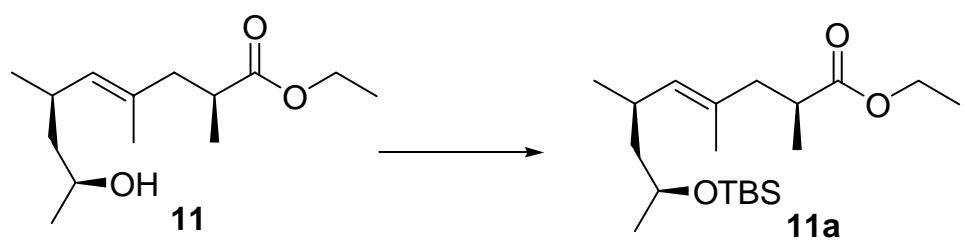

To a solution of alcohol $11(475 \mathrm{mg}, 1.96 \mathrm{mmol})$ in $\mathrm{CH}_{2} \mathrm{Cl}_{2}(6 \mathrm{~mL})$ was added $\mathrm{Et}_{3} \mathrm{~N}$ $(0.41 \mathrm{~mL}, 2.94 \mathrm{mmol})$ and TBSOTf $(0.54 \mathrm{~mL}, 2.35 \mathrm{mmol})$ at room temperature. After stirring for $3 \mathrm{~h}$, the reaction mixture was quenched with $\mathrm{NH}_{4} \mathrm{Cl}$ (sat) and extracted with EtOAc. The organic extracts were dried over anhydrous $\mathrm{MgSO}_{4}$, filtered, and concentrated in vacuo. Flash chromatography on silica gel (3\% ethyl acetate in hexane) produced $685 \mathrm{mg}(1.92 \mathrm{mmol}, 98 \%)$ of 11a, $[\alpha]_{\mathrm{D}}{ }^{23}=-7.3, c$ 2.0, $\mathrm{CHCl}_{3}$; IR (neat) $1737 ;{ }^{1} \mathrm{H} \mathrm{NMR}\left(400 \mathrm{MHz}, \mathrm{CDCl}_{3}\right) \delta 4.92(\mathrm{~d}, J=$ 9.6 Hz, $1 \mathrm{H}), 4.10-4.05(\mathrm{~m}, 2 \mathrm{H}), 3.75-3.70(\mathrm{~m}, 1 \mathrm{H}), 2.59-2.53(\mathrm{~m}, 1 \mathrm{H}), 2.44-2.38(\mathrm{~m}, 1 \mathrm{H}), 2.35-$ 2.30(m, $1 \mathrm{H}), 2.01-1.95(\mathrm{~m}, 1 \mathrm{H}), 1.56(\mathrm{~d}, J=1.1 \mathrm{~Hz}, 3 \mathrm{H}), 1.42-1.36(\mathrm{~m}, 1 \mathrm{H}), 1.29-1.24(\mathrm{~m}, 1 \mathrm{H})$, 
1.22(t, $3 \mathrm{H}), 1.07-1.05(\mathrm{~m}, 6 \mathrm{H}), 0.87-0.86(\mathrm{~m}, 12 \mathrm{H}), 0.02(\mathrm{~s}, 6 \mathrm{H}) ;{ }^{13} \mathrm{C} \mathrm{NMR}\left(100 \mathrm{MHz}, \mathrm{CDCl}_{3}\right)$ $\delta$ 176.6, 133.9, 130.2, 66.6, 60.1, 47.5, 44.1, 37.8, 29.0, 25.8, 23.6, 20.8, 18.1, 16.4, 15.5, 14.2, $-4.37,-4.77 ;$ LRMS(ESI) $\mathrm{m} / \mathrm{z}[\mathrm{M}+\mathrm{Na}]^{+}$378.87, HRMS(ESI) $[\mathrm{M}+\mathrm{Na}]^{+}$: Calculated for $\mathrm{C}_{20} \mathrm{H}_{40} \mathrm{O}_{3} \mathrm{Si}: 379.2644$, Found : 379.2648 .

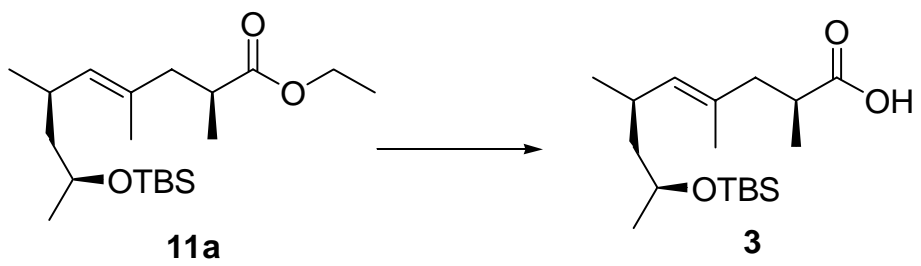

To a solution of 11a (685 mg, $1.92 \mathrm{mmol})$ in THF / $\mathrm{H}_{2} \mathrm{O} / \mathrm{MeOH}(3 \mathrm{~mL} / 1 \mathrm{~mL} / 1 \mathrm{~mL})$ was added $\mathrm{LiOH}(242 \mathrm{mg}, 5.76 \mathrm{mmol})$ at room temperature. After stirring for $6 \mathrm{~h}$, the reaction mixture was quenched with $1 \mathrm{~N} \mathrm{HCl}(\mathrm{aq})$ until $\mathrm{pH} 3$ and extracted with EtOAc. The organic extracts were dried over anhydrous $\mathrm{MgSO}_{4}$, filtered, and concentrated in vacuo. Flash chromato graphy on silica gel (10\% ethyl acetate in hexane) produced $599 \mathrm{mg}(1.82 \mathrm{mmol}, 95 \%)$ of $\mathbf{3}$;

${ }^{1} \mathrm{H}$ NMR $\left(400 \mathrm{MHz}, \mathrm{CDCl}_{3}\right) \delta 4.95(\mathrm{~d}, J=9.4 \mathrm{~Hz}, 1 \mathrm{H}), 3.75-3.70(\mathrm{~m}, 1 \mathrm{H}), 2.62-2.57(\mathrm{~m}, 1 \mathrm{H})$, 2.43-2.34(m, 2 H), 2.03-1.98(m, 1 H), 1.57(d, J = 0.8 Hz, 3 H), 1.41-1.39(m, 1 H), 1.29-1.25(m, $1 \mathrm{H}), 1.08(\mathrm{t}, 6 \mathrm{H}), 0.89-0.86(\mathrm{~m}, 12 \mathrm{H}), 0.02(\mathrm{~s}, 6 \mathrm{H}) ;{ }^{13} \mathrm{C} \mathrm{NMR}\left(100 \mathrm{MHz}, \mathrm{CDCl}_{3}\right) \delta 182.9$, $134.3,129.8,66.7,47.5,43.7,37.7,29.0,25.8,23.6,20.8,18.1,16.1,15.5,-4.38,-4.77$

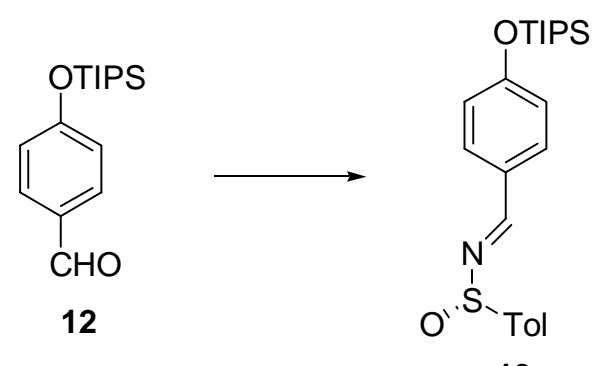

13

To a stirred solution of $\mathbf{1 2}(1.7 \mathrm{~g}, 6 \mathrm{mmol})$ in $\mathrm{CH}_{2} \mathrm{Cl}_{2}(20 \mathrm{~mL})$ was added $(S)$-p-toluene sulfinamide $(950 \mathrm{mg}, 6 \mathrm{mmol})$ and $\mathrm{Ti}(\mathrm{OEt})_{4}(6.4 \mathrm{~mL}, 30 \mathrm{mmol})$ at $0{ }^{\circ} \mathrm{C}$. The reaction mixture was stirred at $40{ }^{\circ} \mathrm{C}$ for $5 \mathrm{~h}$. The reaction mixture was cooled to $0{ }^{\circ} \mathrm{C}$ and was poured into $\mathrm{H}_{2} \mathrm{O}$ 
and was filtered with celite. The reaction mixture was washed with $\mathrm{H}_{2} \mathrm{O}$ and aqueous layer was extracted with $\mathrm{CH}_{2} \mathrm{Cl}_{2}$. and the combined organic extracts were dried over anhydrous $\mathrm{MgSO}_{4}$, filtered, and concentrated in vacuo. Flash chromatography on silica gel (5\% ethyl acetate in hexane) afforded $2.2 \mathrm{~g}(5.3 \mathrm{mmol}, 87 \%)$ of $13 ;{ }^{1} \mathrm{H}$ NMR $\left(400 \mathrm{MHz}, \mathrm{CDCl}_{3}\right) \delta 8.64(\mathrm{~s}, 1 \mathrm{H})$, $7.71(\mathrm{~d}, J=11.2 \mathrm{~Hz}, 1 \mathrm{H}), 7.60(\mathrm{~d}, J=8.2 \mathrm{~Hz}, 1 \mathrm{H}), 7.28(\mathrm{~d}, J=8.1 \mathrm{~Hz}, 1 \mathrm{H}), 6.89(\mathrm{~d}, J=8.7 \mathrm{~Hz}$, $1 \mathrm{H}), 2.37(\mathrm{~s}, 3 \mathrm{H}), 1.27-1.21(\mathrm{~m}, 3 \mathrm{H}), 1.09-1.03(\mathrm{~m}, 18 \mathrm{H})$

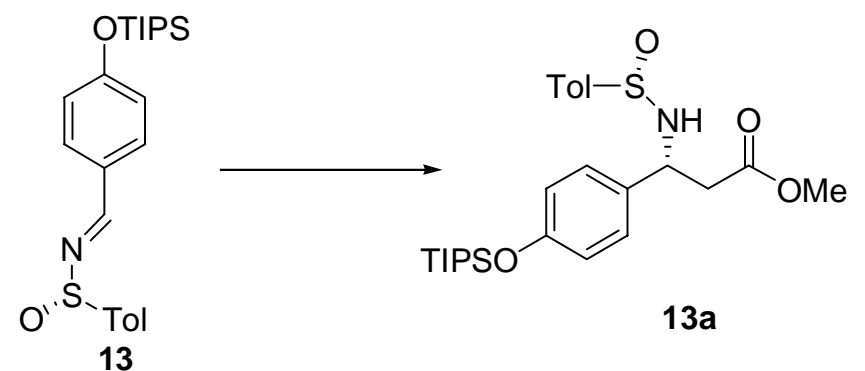

To a stirred solution of distilled methylacetate $(1 \mathrm{~mL}, 12.5 \mathrm{mmol})$ in $\mathrm{Et}_{2} \mathrm{O}(10 \mathrm{~mL})$ was added NaHMDS $(12.5 \mathrm{~mL}, 12.5 \mathrm{mmol})$ at $-78{ }^{\circ} \mathrm{C}$. The reaction mixture was stirred for 40 min. Compound 13 (1.3 g, $3.1 \mathrm{mmol})$ was added to the enolate mixture at the same temperature. The resulting mixture was stirred for $1 \mathrm{~h}$. The reaction mixture was poured into $\mathrm{NH}_{4} \mathrm{Cl}$ (sat) and the aqueous layer was extracted with EtOAc and the combined organic extracts were dried over anhydrous $\mathrm{MgSO}_{4}$, filtered, and concentrated in vacuo. Flash chromatography on silica gel (35\% ethyl acetate in hexane) afforded $1.2 \mathrm{~g}(2.5 \mathrm{mmol}, 78 \%)$ of 13a, $[\alpha]_{\mathrm{D}}^{23}=+37.5, c 1.0$, $\mathrm{CHCl}_{3}$; IR (neat) $3181,1739,1607,1265,1169 ;{ }^{1} \mathrm{H} \mathrm{NMR}\left(400 \mathrm{MHz}, \mathrm{CDCl}_{3}\right) \delta 7.56(\mathrm{~d}, J=8.2$ $\mathrm{Hz}, 1 \mathrm{H}), 7.28-7.22(\mathrm{~m}, 4 \mathrm{H}), 6.85(\mathrm{~d}, J=8.4 \mathrm{~Hz}, 1 \mathrm{H}), 4.88-4.82(\mathrm{~m}, 2 \mathrm{H}), 3.56(\mathrm{~s}, 3 \mathrm{H}), 2.78(\mathrm{~d}, J$ $=6.3 \mathrm{~Hz}, 2 \mathrm{H}), 2.39(\mathrm{~s}, 3 \mathrm{H}), 1.27-1.20(\mathrm{~m}, 3 \mathrm{H}), 1.12-1.06(\mathrm{~m}, 18 \mathrm{H}) ;{ }^{13} \mathrm{C} \mathrm{NMR}(100 \mathrm{MHz}$, $\left.\mathrm{CDCl}_{3}\right) \delta 171.3,155.8,142.3,141.2,132.3,129.4,128.3,125.2,120.0$ 54.2, 51.7, 42.0, 21.2, 17.8, 12.5 ; LRMS (ESI) $\mathrm{m} / \mathrm{z}[\mathrm{M}+\mathrm{Na}]^{+}$512.05, HRMS (ESI) $[\mathrm{M}+\mathrm{Na}]^{+}$: Calculated for $\mathrm{C}_{26} \mathrm{H}_{39} \mathrm{NO}_{4} \mathrm{SSi}$ : 512.2267 , Found : 512.2261. 


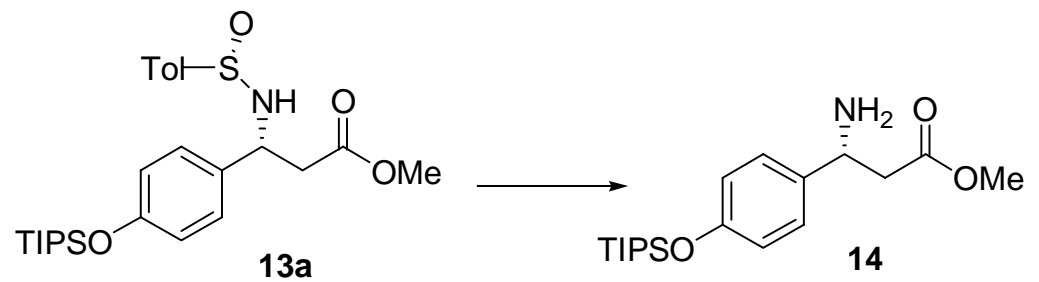

To a stirred solution of 13a $(1.1 \mathrm{~g}, 2.3 \mathrm{mmol})$ in $\mathrm{MeOH}(5 \mathrm{~mL})$ was added Trifluoroacetic acid $(0.34 \mathrm{~mL} 4.6 \mathrm{mmol})$ at $0^{\circ} \mathrm{C}$. The reaction mixture was stirred for $1 \mathrm{~h}$. The reaction mixture was poured into $\mathrm{NaHCO}_{3}$ (sat) and $\mathrm{pH}$ adjusted to 7-8 and aqueous layer was extracted with $\mathrm{CH}_{2} \mathrm{Cl}_{2}$ and the combined organic extracts were dried over anhydrous $\mathrm{MgSO}_{4}$, filtered, and concentrated in vacuo. Flash chromatography on silica gel $(5 \%$ methanol in dichloromethane) afforded $653 \mathrm{mg}(1.9 \mathrm{mmol}, 83 \%)$ of $\mathbf{1 4},[\alpha]_{\mathrm{D}}{ }^{23}=+7.1, c 2.4, \mathrm{CHCl}_{3}$; IR (neat) $1737,1607,1510,1265 ;{ }^{1} \mathrm{H}$ NMR $\left(400 \mathrm{MHz}, \mathrm{CDCl}_{3}\right) \delta 7.16(\mathrm{~d}, J=8.4 \mathrm{~Hz}, 1 \mathrm{H}), 6.81(\mathrm{~d}$, $J=8.6 \mathrm{~Hz}, 1 \mathrm{H}), 4.33(\mathrm{t}, 1 \mathrm{H}), 3.64(\mathrm{~s}, 3 \mathrm{H}), 2.60(\mathrm{~d}, J=6.9 \mathrm{~Hz}, 2 \mathrm{H}), 1.24-1.18(\mathrm{~m}, 3 \mathrm{H}), 1.07-$ $1.05(\mathrm{~m}, 18 \mathrm{H}) ;{ }^{13} \mathrm{C} \mathrm{NMR}\left(100 \mathrm{MHz}, \mathrm{CDCl}_{3}\right) \delta 172.5,155.1,136.9,126.9,119.8,51.9,51.5$, 44.0, 17.8, $12.5 ;$ LRMS(ESI) $\mathrm{m} / \mathrm{z}[\mathrm{M}+\mathrm{Na}]^{+}$373.67, HRMS(ESI) $[\mathrm{M}+\mathrm{Na}]^{+}$: Calculated for $\mathrm{C}_{26} \mathrm{H}_{39} \mathrm{NO}_{4} \mathrm{SSi}$ : 374.2127 , Found : 374.2126 .<smiles>CC(NC(C)(C)OC(C)(C)C)C(=O)N(C)[C@H](Cc1c(Br)[nH]c2ccccc12)C(=O)O</smiles>

15

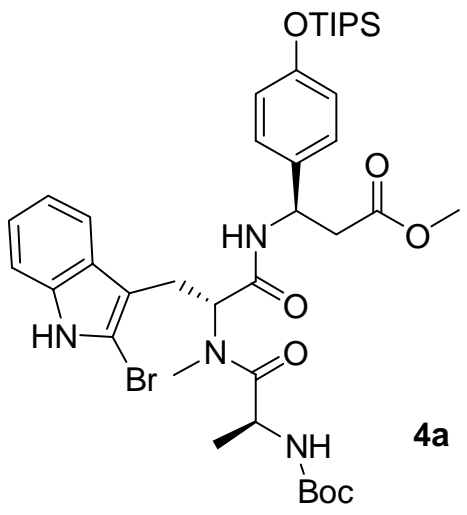

To a stirred solution of acid 15 (49 $\mathrm{mg}, 0.1 \mathrm{mmol})$ in THF ( $2 \mathrm{~mL}$ ) was added amine $\mathbf{1 4}$ (36 $\mathrm{mg}, 0.1 \mathrm{mmol})$ and DCC $(23 \mathrm{mg}, 0.11 \mathrm{mmol})$ at $0{ }^{\circ} \mathrm{C}$. The reaction mixture was stirred for $12 \mathrm{~h}$. The reaction mixture was filtered and concentrated in vacuo. Flash chromatography on silica gel (35\% ethyl acetate in hexane) afforded $66 \mathrm{mg}(0.083 \mathrm{mmol}, 83 \%)$ of $4 \mathbf{a},[\alpha]_{\mathrm{D}}{ }^{23}=+$ 20.3, c 2.0, $\mathrm{CHCl}_{3}$; IR (neat) 3323, 1652, 1511, 1451, $1267 ;{ }^{1} \mathrm{H}$ NMR (400 MHz, $\left.\mathrm{CDCl}_{3}\right) \delta$ 8.15(s, $1 \mathrm{H}), 7.50(\mathrm{~d}, J=7.7 \mathrm{~Hz}, 1 \mathrm{H}), 7.19(\mathrm{~d}, J=7.8 \mathrm{~Hz}, 1 \mathrm{H}), 7.12-7.03(\mathrm{~m}, 4 \mathrm{H}), 6.84(\mathrm{~d}, J=$ 
$8.3 \mathrm{~Hz}, 1 \mathrm{H}), 6.76(\mathrm{~d}, J=8.5 \mathrm{~Hz}, 2 \mathrm{H}), 5.67-5.63(\mathrm{~m}, 1 \mathrm{H}), 5.40-5.35(\mathrm{~m}, 1 \mathrm{H}), 5.08(\mathrm{~d}, J=6.8 \mathrm{~Hz}$, $1 \mathrm{H}), 4.30-4.24(\mathrm{~m}, 1 \mathrm{H}), 3.57(\mathrm{~s}, 3 \mathrm{H}), 3.36(\mathrm{dd}, J=15.3 \mathrm{~Hz}, 5.4 \mathrm{~Hz}, 1 \mathrm{H}), 3.19(\mathrm{dd}, J=15.2 \mathrm{~Hz}$, $11.0 \mathrm{~Hz}, 1 \mathrm{H}), 2.94(\mathrm{~s}, 3 \mathrm{H}), 2.84(\mathrm{dd}, J=15.2 \mathrm{~Hz}, 7.5 \mathrm{~Hz}, 1 \mathrm{H}), 2.73(\mathrm{dd}, J=15.2 \mathrm{~Hz}, 5.8 \mathrm{~Hz}, 1$ $\mathrm{H}), 1.34(\mathrm{~s}, 9 \mathrm{H}), 1.25-1.18(\mathrm{~m}, 3 \mathrm{H}), 1.07-1.04(\mathrm{~m}, 18 \mathrm{H}), 0.63(\mathrm{~d}, J=6.8 \mathrm{~Hz}, 3 \mathrm{H}) ;{ }^{13} \mathrm{C} \mathrm{NMR}$ $\left(100 \mathrm{MHz}, \mathrm{CDCl}_{3}\right) \delta 174.1,171.1,169.1,155.5,136.0,132.8,127.4,127.3,122.4,120.1,119.9$, 118.3, 110.8, 110.3, 108.9, 79.6, 56.0, 51.7, 49.4, 46.4, 40.6, 31.5, 28.3, 23.3, 17.9, 16.8, 12.6 ; LRMS(ESI) $\mathrm{m} / \mathrm{z}[\mathrm{M}+\mathrm{Na}]^{+}$823.0, HRMS(ESI) $[\mathrm{M}+\mathrm{Na}]^{+}$: Calculated for $\mathrm{C}_{26} \mathrm{H}_{39} \mathrm{NO}_{4} \mathrm{SSi}$ : 823.3078, Found : 823.3086.
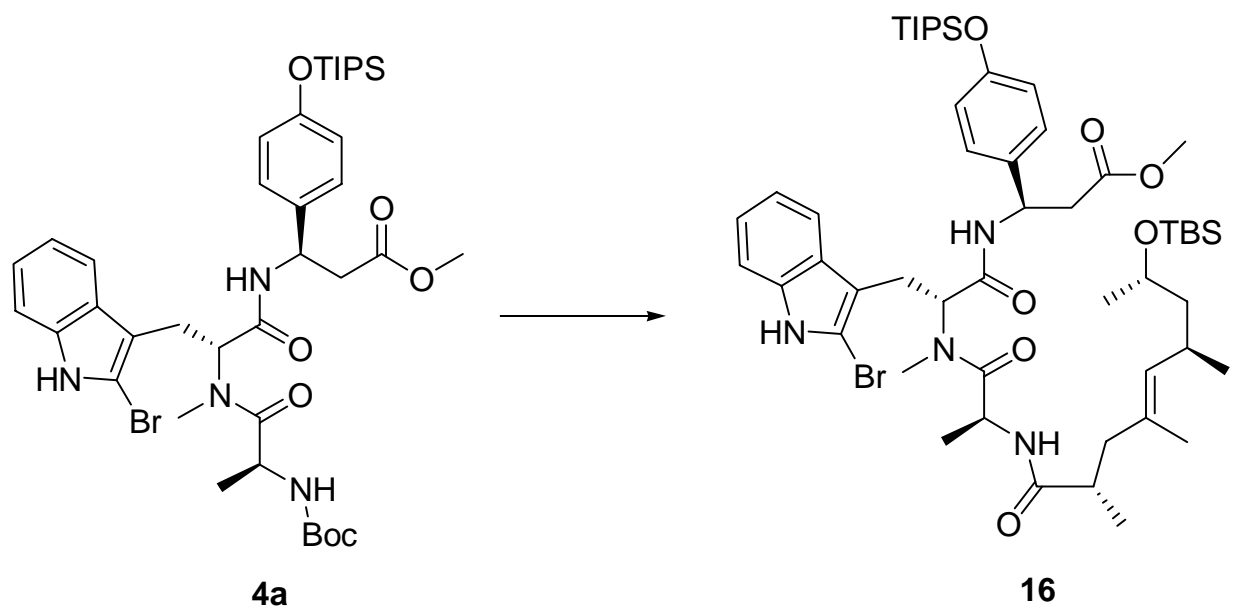

To a stirred solution of $4 \mathbf{a}(121 \mathrm{mg}, 0.15 \mathrm{mmol})$ in $\mathrm{CH}_{2} \mathrm{Cl}_{2}(1 \mathrm{~mL})$ was trifluoroacetic acid $(0.5 \mathrm{~mL})$ at $0{ }^{\circ} \mathrm{C}$. The reaction mixture was stirred for $1 \mathrm{~h}$. The reaction mixture was poured into $\mathrm{NaHCO}_{3}$ (sat) and $\mathrm{pH}$ adjusted to 7-8 and aqueous layer was extracted with $\mathrm{CH}_{2} \mathrm{Cl}_{2}$. The combined organic extracts were dried over anhydrous $\mathrm{MgSO}_{4}$, filtered, and concentrated in vacuo. Flash chromatography on silica gel (5\% methanol in dichloromethane) afforded $100 \mathrm{mg}$ (0.14 mmol, $94 \%)$ of 4.

To a stirred solution of amine $4(50 \mathrm{mg}, 0.07 \mathrm{mmol})$ in THF $(2 \mathrm{~mL})$ was added acid $\mathbf{3}$ (22.4 mg, $0.07 \mathrm{mmol})$, DCC (15 mg, $0.074 \mathrm{mmol})$ and HOBT (9.2 mg, $0.07 \mathrm{mmol})$ at $-20{ }^{\circ} \mathrm{C}$. The reaction mixture was warmed to $0{ }^{\circ} \mathrm{C}$ and was stirred for $12 \mathrm{~h}$. The reaction was stirred at room temperature for additional $12 \mathrm{~h}$. The reaction mixture was filtered and concentrated in 
vacuo. Flash chromatography on silica gel (35\% ethyl acetate in hexane) afforded $55.3 \mathrm{mg}$ $(0.056 \mathrm{mmol}, 80 \%)$ of 16, $[\alpha]_{\mathrm{D}}{ }^{23}=+18.3, c 1.0, \mathrm{CHCl}_{3}$; IR (neat) $3303,1742,1641,1511$, 1451, $1267 ;{ }^{1} \mathrm{H}$ NMR $\left(400 \mathrm{MHz}, \mathrm{CDCl}_{3}\right) \delta 8.59(\mathrm{~s}, 1 \mathrm{H}), 7.50(\mathrm{~d}, J=7.6 \mathrm{~Hz}, 1 \mathrm{H}), 7.20(\mathrm{~d}, J=$ $7.8 \mathrm{~Hz}, 1 \mathrm{H}), 7.12-7.04(\mathrm{~m}, 4 \mathrm{H}), 6.98(\mathrm{~d}, J=8.5 \mathrm{~Hz}, 1 \mathrm{H}), 6.76(\mathrm{~d}, J=8.4 \mathrm{~Hz}, 2 \mathrm{H}), 6.11(\mathrm{~d}, J=$ $5.7 \mathrm{~Hz}, 1 \mathrm{H}), 5.63-5.59(\mathrm{~m}, 1 \mathrm{H}), 5.40-5.34(\mathrm{~m}, 1 \mathrm{H}), 4.91(\mathrm{~d}, J=9.3 \mathrm{~Hz}, 1 \mathrm{H}), 4.50-4.45(\mathrm{~m}, 1 \mathrm{H})$, 3.74-3.69(m, 1 H), 3.56(s, 3 H), 3.39(dd, $J=15.4 \mathrm{~Hz}, 5.5 \mathrm{~Hz}, 1 \mathrm{H}), 3.18(\mathrm{dd}, J=15.4 \mathrm{~Hz}, 10.9$ $\mathrm{Hz}, 1 \mathrm{H}), 2.97(\mathrm{~s}, 3 \mathrm{H}), 2.89(\mathrm{dd}, J=15.4 \mathrm{~Hz}, 7.8 \mathrm{~Hz}, 1 \mathrm{H}), 2.75(\mathrm{dd}, J=15.4 \mathrm{~Hz}, 5.8 \mathrm{~Hz}, 1 \mathrm{H})$, 2.43-2.38(m, $1 \mathrm{H}), 2.33-2.24(\mathrm{~m}, 2 \mathrm{H}), 1.94-1.89(\mathrm{~m}, 1 \mathrm{H}), 1.51(\mathrm{~d}, J=1.0 \mathrm{~Hz}, 3 \mathrm{H}), 1.26-1.18(\mathrm{~m}$, $5 \mathrm{H}), 1.07-1.05(\mathrm{~m}, 21 \mathrm{H}), 0.98(\mathrm{~d}, J=6.8 \mathrm{~Hz}, 3 \mathrm{H}), 0.85-0.84(\mathrm{~m}, 12 \mathrm{H}), 0.69(\mathrm{~d}, J=6.8 \mathrm{~Hz}, 3 \mathrm{H})$, 0.064(s, $6 \mathrm{H}) ;{ }^{13} \mathrm{C}$ NMR $\left(100 \mathrm{MHz}, \mathrm{CDCl}_{3}\right) \delta 176.1,173.7,171.2,168.7,155.3,135.9,134.2$, 132.8, 129.9, 127.4, 122.3, 120.1, 119.8, 118.2, 110.8, 110.2, 108.8, 66.6, 56.2, 51.6, 49.4, 47.5, $45.4,43.8,40.6,38.3,33.9,28.9,25.8,24.9,23.6,20.7,18.0,17.8,16.5,16.2,15.4,12.5,-4.43$, $-4.85 ;$ LRMS(ESI) $\mathrm{m} / \mathrm{z} \quad[\mathrm{M}+\mathrm{Na}]^{+}$1033.42, HRMS(ESI) $[\mathrm{M}+\mathrm{Na}]^{+}:$Calculated for $\mathrm{C}_{52} \mathrm{H}_{83} \mathrm{BrN}_{4} \mathrm{O}_{7} \mathrm{Si}_{2}: 1033.4881$, Found :1033.4885.

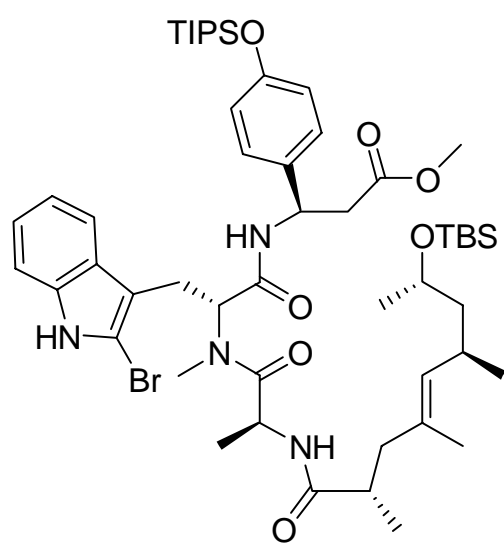

16

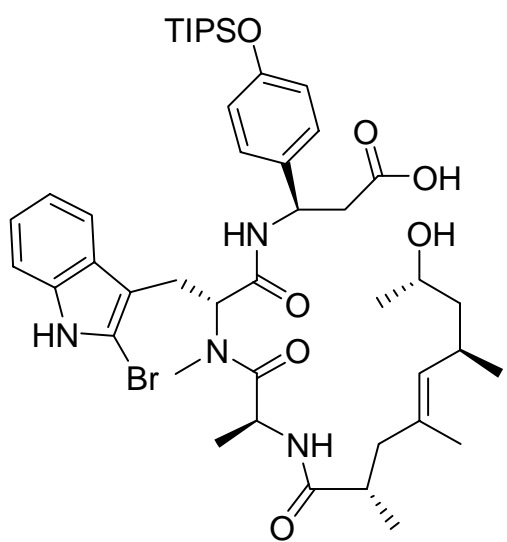

2

To a solution of $16(51 \mathrm{mg}, 0.05 \mathrm{mmol})$ in $\mathrm{THF} / \mathrm{H}_{2} \mathrm{O} / \mathrm{MeOH}(3 \mathrm{~mL} / 1 \mathrm{~mL} / 1 \mathrm{~mL})$ was added $\mathrm{LiOH}(6.3 \mathrm{mg}, 0.15 \mathrm{mmol})$ at room temperature. After stirring for $2 \mathrm{~h}$, the reaction mixture was quenched with $1 \mathrm{~N} \mathrm{HCl}(\mathrm{aq})$ until $\mathrm{pH} 3$ and extracted with EtOAc. The organic 
extracts were dried over anhydrous $\mathrm{MgSO}_{4}$, filtered, and concentrated in vacuo. Flash chromatography on silica gel (65\% ethyl acetate in hexane) produced $40 \mathrm{mg}(0.048 \mathrm{mmol}, 96 \%)$ of free phenol-acid compound.

To a solution of free phenol-acid compound $(12 \mathrm{mg}, 0.014 \mathrm{mmol})$ in $\mathrm{CH}_{2} \mathrm{Cl}_{2}(1 \mathrm{~mL})$ was added 2,6-lutidine $(7 \mu \mathrm{L}, 0.056 \mathrm{mmol})$ and TIPSOTf $(12 \mu \mathrm{L}, 0.042 \mathrm{mmol})$ at room temperature. After stirring for $1 \mathrm{~h}$, the reaction mixture was quenched with $\mathrm{H}_{2} \mathrm{O}$ and extracted with $\mathrm{CH}_{2} \mathrm{Cl}_{2}$. The organic extracts were dried over anhydrous $\mathrm{MgSO}_{4}$, filtered, and concentrated in vacuo.

To a solution of above crude compound in THF / $\mathrm{H}_{2} \mathrm{O} / \mathrm{MeOH}(0.4 \mathrm{~mL} / 0.2 \mathrm{~mL} / 0.2$ $\mathrm{mL}$ ) was added $\mathrm{K}_{2} \mathrm{CO}_{3}(4 \mathrm{mg}, 0.03 \mathrm{mmol})$ at room temperature. After stirring for $30 \mathrm{~min}, 2 \mathrm{~N}$ $\mathrm{HCl}(\mathrm{aq})$ was added to the reaction mixture and was stirred for $1 \mathrm{~h}$. The reaction mixture was extracted with EtOAc. The organic extracts were dried over anhydrous $\mathrm{MgSO}_{4}$, filtered, and concentrated in vacuo. Flash chromatography on silica gel (5\% methanol in dichloromethane) produced $10 \mathrm{mg}(0.0112 \mathrm{mmol}, 80 \%)$ of seco acid $2,[\alpha]_{\mathrm{D}}^{23}=+32, c 0.8, \mathrm{CHCl}_{3}$; IR (neat) 3295 , 2475, 1717, 1640, 1511, 1449, $1267 ;{ }^{1} \mathrm{H}$ NMR (400 MHz, $\left.\mathrm{CD}_{3} \mathrm{OD}\right) \delta 7.48(\mathrm{~d}, J=7.8 \mathrm{~Hz}, 1 \mathrm{H})$, 7.22(d, $J=7.1 \mathrm{~Hz}, 3 \mathrm{H}), 7.05(\mathrm{t}, 1 \mathrm{H}), 6.97(\mathrm{t}, 1 \mathrm{H}), 6.76(\mathrm{~d}, J=8.6 \mathrm{~Hz}, 2 \mathrm{H}), 5.69-5.65(\mathrm{~m}, 1 \mathrm{H})$, 5.46-5.42(m, $1 \mathrm{H}), 4.93(\mathrm{~d}, J=9.6 \mathrm{~Hz}, 1 \mathrm{H}), 4.40-4.35(\mathrm{~m}, 1 \mathrm{H}), 3.69-3.64(\mathrm{~m}, 1 \mathrm{H}), 3.46(\mathrm{dd}, J=$ $15.2 \mathrm{~Hz}, 4.5 \mathrm{~Hz}, 1 \mathrm{H}), 3.13(\mathrm{dd}, J=15.2 \mathrm{~Hz}, 11.8 \mathrm{~Hz}, 1 \mathrm{H}), 3.07(\mathrm{~s}, 3 \mathrm{H}), 2.92(\mathrm{dd}, J=15.8 \mathrm{~Hz}$, $8.8 \mathrm{~Hz}, 1 \mathrm{H}), 2.77(\mathrm{dd}, J=15.8 \mathrm{~Hz}, 5.1 \mathrm{~Hz}, 1 \mathrm{H}), 2.48-2.42(\mathrm{~m}, 2 \mathrm{H}), 2.27-2.23(\mathrm{~m}, 1 \mathrm{H}), 1.92-$ 1.87(m, 1 H), 1.56(s, 3 H), 1.45-1.39(m, 1 H), 1.28-1.20(m, 4 H), 1.10-1.06(m, 21 H), 0.97(d, $J$ $=6.8 \mathrm{~Hz}, 3 \mathrm{H}), 0.90(\mathrm{~d}, J=6.5 \mathrm{~Hz}, 3 \mathrm{H}), 0.62(\mathrm{~d}, J=6.9 \mathrm{~Hz}, 3 \mathrm{H}) ;{ }^{13} \mathrm{C} \mathrm{NMR}(100 \mathrm{MHz}$, $\left.\mathrm{CD}_{3} \mathrm{OD}\right) \delta 179.2,176.2,174.7,171.2,156.6,137.9,135.5,134.8,131.9,128.9,128.7,122.7$, 120.8, 120.4, 119.0, 111.5, 110.7, 110.3, 66.6, 57.9, 51.1, 46.9, 44.9, 41.8, 38.9, 32.8, 30.8, 30.4, 24.4, 23.4, 21.4, 18.4, 16.9, 16.0, 15.6, 13.9 ; LRMS(ESI) m/z [M+Na $]^{+}$905.29, HRMS(ESI) $[\mathrm{M}+\mathrm{Na}]^{+}$: Calculated for $\mathrm{C}_{45} \mathrm{H}_{67} \mathrm{BrN}_{4} \mathrm{O}_{7} \mathrm{Si}: 905.3860$, Found : 905.3872. 

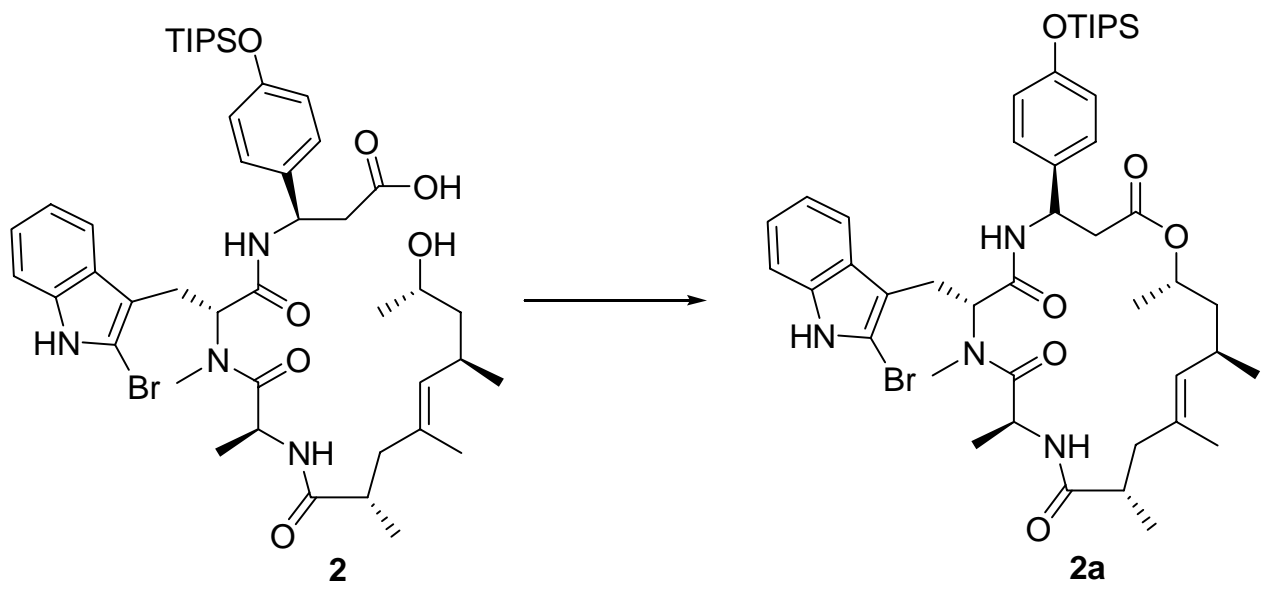

To a solution of seco acid 2 (10 mg, $0.011 \mathrm{mmol})$ in THF $(2 \mathrm{~mL})$ was added DIPEA (20 $\mu \mathrm{L}, 0.11 \mathrm{mmol}$ ) and 2,4,6-trichlorobenzoyl chloride $(9 \mu \mathrm{L}, 0.055 \mathrm{mmol}$ ) at room temperature. After stirring for $2.5 \mathrm{~h}$, the reaction mixture was concentrated in vacuo. The reaction mixture was diluted with dry benzene $(100 \mathrm{~mL})$. To a solution of the resulting mixture was slowly added DMAP (7 mg, $0.055 \mathrm{mmol})$ in benzene $(10 \mathrm{~mL})$ over a period of $8 \mathrm{~h}$. The reaction mixture was concentrated in vacuo and then was directly purified by column chromatography on silica gel (35\% ethyl acetate in hexane) to gave $8 \mathrm{mg}(0.009 \mathrm{mmol}, 82 \%)$ of 2a, $[\alpha]_{\mathrm{D}}^{23}=+58, c 0.7, \mathrm{CHCl}_{3}$; IR (neat) $3305,1641,1510,1455,1266 ;{ }^{1} \mathrm{H} \mathrm{NMR}(400 \mathrm{MHz}$, $\left.\mathrm{CDCl}_{3}\right) \delta 8.42(\mathrm{~s}, 1 \mathrm{H}), 7.53(\mathrm{~d}, J=7.1 \mathrm{~Hz}, 1 \mathrm{H}), 7.48(\mathrm{~d}, J=9.1 \mathrm{~Hz}, 1 \mathrm{H}), 7.21(\mathrm{~d}, J=8.4 \mathrm{~Hz}, 1$ H), 7.12-7.02(m, $2 \mathrm{H}), 7.01(\mathrm{~d}, J=8.6 \mathrm{~Hz}, 2 \mathrm{H}), 6.77(\mathrm{~d}, J=8.6 \mathrm{~Hz}, 2 \mathrm{H}), 6.60(\mathrm{~d}, J=6.5 \mathrm{~Hz}, 1$ H), 5.84-5.80(m, $1 \mathrm{H}), 5.31-5.26(\mathrm{~m}, 1 \mathrm{H}), 4.71-4.65(\mathrm{~m}, 2 \mathrm{H}), 4.59-4.54(\mathrm{~m}, 1 \mathrm{H}), 3.31(\mathrm{dd}, J=$ $15.2 \mathrm{~Hz}, 6.2 \mathrm{~Hz}, 1 \mathrm{H}), 3.23(\mathrm{dd}, J=15.2 \mathrm{~Hz}, 10.6 \mathrm{~Hz}, 1 \mathrm{H}), 2.93(\mathrm{~s}, 3 \mathrm{H}), 2.65(\mathrm{dd}, J=14.8 \mathrm{~Hz}$, $4.8 \mathrm{~Hz}, 1 \mathrm{H}), 2.58(\mathrm{dd}, J=14.8 \mathrm{~Hz}, 4.9 \mathrm{~Hz}, 1 \mathrm{H}), 2.49-2.34(\mathrm{~m}, 2 \mathrm{H}), 2.23-2.19(\mathrm{~m}, 1 \mathrm{H}), 1.84(\mathrm{~d}$, $J=15.6 \mathrm{~Hz}, 1 \mathrm{H}), 1.54(\mathrm{~s}, 3 \mathrm{H}), 1.26-1.19(\mathrm{~m}, 4 \mathrm{H}), 1.10-1.04(\mathrm{~m}, 22 \mathrm{H}), 1.02(\mathrm{~d}, J=6.3 \mathrm{~Hz}, 3 \mathrm{H})$, $0.78(\mathrm{~d}, J=6.5 \mathrm{~Hz}, 3 \mathrm{H}), 0.66(\mathrm{~d}, J=6.7 \mathrm{~Hz}, 3 \mathrm{H}) ;{ }^{13} \mathrm{C} \mathrm{NMR}\left(100 \mathrm{MHz}, \mathrm{CDCl}_{3}\right) \delta 174.7,174.3$, 170.7, 168.8, 155.4, 136.1, 133.6, 132.1, 127.3, 127.1, 122.4, 120.1, 119.8, 118.2, 110.4, 108.9, 70.5, 55.1, 48.7, 45.9, 43.4, 40.5, 39.9, 39.2, 30.7, 29.1, 23.0, 21.9, 20.4, 18.9, 18.5, 17.9, 17.6, $12.6 ; \operatorname{LRMS}(\mathrm{ESI}) \quad \mathrm{m} / \mathrm{z} \quad[\mathrm{M}+\mathrm{Na}]^{+}$887.31, $\mathrm{HRMS}(\mathrm{ESI}) \quad[\mathrm{M}+\mathrm{Na}]^{+}$: calculated for $\mathrm{C}_{45} \mathrm{H}_{65} \mathrm{BrN}_{4} \mathrm{O}_{6} \mathrm{Si}: 887.3754$, Found : 887.3760. 


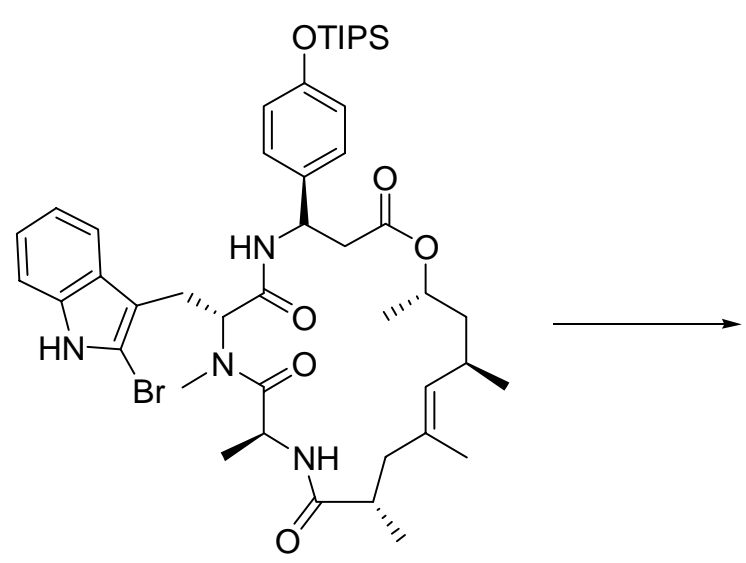

$2 a$

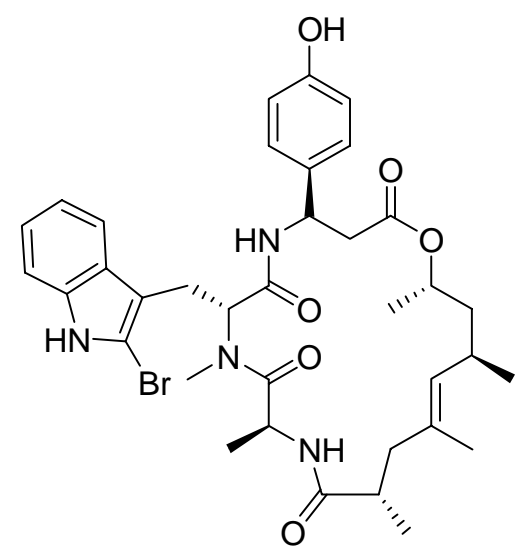

(+)-Jasplakinolide (1)

To a stirred solution of $\mathbf{2 a}(3 \mathrm{mg}, 3.5 \mu \mathrm{mol})$ in THF $(1 \mathrm{~mL})$ was added TBAF $(0.1 \mathrm{M}$ solution, $35 \mu \mathrm{L}, 3.5 \mu \mathrm{mol})$ at $0{ }^{\circ} \mathrm{C}$. The reaction mixture was stirred for $10 \mathrm{~min}$. The reaction mixture was poured into $\mathrm{NH}_{4} \mathrm{Cl}$ (sat) and the aqueous layer was extracted with EtOAc and the combined organic extracts were dried over anhydrous $\mathrm{MgSO}_{4}$, filtered, and concentrated in vacuo. Flash chromatography on silica gel (50\% ethyl acetate in hexane) produced (+)Jasplakinolide (1) $(2.2 \mathrm{mg}, 90 \%),[\alpha]_{\mathrm{D}}^{23}=+67.7, c 0.2, \mathrm{CH}_{2} \mathrm{Cl}_{2}$; IR (neat) $3305,1715,1684$, 1675, 1640, 1515, $1450 ;{ }^{1} \mathrm{H}$ NMR (400 MHz, $\left.\mathrm{CDCl}_{3}\right) \delta 8.36(\mathrm{~s}, 1 \mathrm{H}), 7.59(\mathrm{~d}, J=8.8 \mathrm{~Hz}, 1 \mathrm{H})$, 7.57(d, $J=7.7 \mathrm{~Hz}, 1 \mathrm{H}), 7.25(\mathrm{~d}, J=8.2 \mathrm{~Hz}, 1 \mathrm{H}), 7.17-7.09(\mathrm{~m}, 2 \mathrm{H}), 6.97(\mathrm{~d}, J=8.4 \mathrm{~Hz}, 2 \mathrm{H})$, $6.68(\mathrm{~d}, J=8.4 \mathrm{~Hz}, 2 \mathrm{H}), 6.61(\mathrm{~d}, J=6.6 \mathrm{~Hz}, 1 \mathrm{H}), 6.19(\mathrm{bs}, 1 \mathrm{H}), 5.87-5.83(\mathrm{~m}, 1 \mathrm{H}), 5.29-5.25(\mathrm{~m}$, $1 \mathrm{H}), 4.76-4.70(\mathrm{~m}, 2 \mathrm{H}), 4.64-4.59(\mathrm{~m}, 1 \mathrm{H}), 3.39(\mathrm{dd}, J=15.3 \mathrm{~Hz}, 6.3 \mathrm{~Hz}, 1 \mathrm{H}), 3.24(\mathrm{dd}, J=$ $15.3 \mathrm{~Hz}, 10.4 \mathrm{~Hz}, 1 \mathrm{H}), 2.98(\mathrm{~s}, 3 \mathrm{H}), 2.68(\mathrm{dd}, J=15.8 \mathrm{~Hz}, 4.6 \mathrm{~Hz}, 1 \mathrm{H}), 2.61(\mathrm{dd}, J=15.8 \mathrm{~Hz}$, $5.6 \mathrm{~Hz}, 1 \mathrm{H}), 2.52-2.46(\mathrm{~m}, 1 \mathrm{H}), 2.42-2.35(\mathrm{~m}, 1 \mathrm{H}), 2.27-2.19(\mathrm{~m}, 1 \mathrm{H}), 1.89(\mathrm{~d}, J=15.7 \mathrm{~Hz}, 1$ H), $1.57(\mathrm{~s}, 3 \mathrm{H}), 1.35-1.28(\mathrm{~m}, 1 \mathrm{H}), 1.12(\mathrm{~d}, J=6.8 \mathrm{~Hz}, 3 \mathrm{H}), 1.06(\mathrm{~d}, J=6.3 \mathrm{~Hz}, 3 \mathrm{H}), 0.81(\mathrm{~d}, J$ $=6.6 \mathrm{~Hz}, 3 \mathrm{H}), 0.72(\mathrm{~d}, J=6.7 \mathrm{~Hz}, 3 \mathrm{H}) ;{ }^{13} \mathrm{C} \mathrm{NMR}\left(100 \mathrm{MHz}, \mathrm{CDCl}_{3}\right) \delta 175.0,174.4,170.8$, 168.9, 155.4, 136.1, 133.6, 131.6, 127.6, 127.2, 122.5, 120.2, 118.1, 115.5, 110.5, 110.3, 108.9, 70.7, 55.4, 48.9, 45.9, 43.3, 40.7, 40.0, 39.6, 30.8, 29.2, 23.2, 21.9, 20.4, 19.0, 18.5, 17.7 ; LRMS(ESI) $\mathrm{m} / \mathrm{z}[\mathrm{M}+\mathrm{Na}]^{+}$731.20, HRMS(ESI) $[\mathrm{M}+\mathrm{Na}]^{+}$: Calculated for $\mathrm{C}_{36} \mathrm{H}_{45} \mathrm{BrN}_{4} \mathrm{O}_{6}$ : 731.2420 , Found : 731.2419 . 